\title{
PAPILOMA INVERTIDO - UM RELATO DE CASO
}

\section{ARTIGO ORIGINAL}

PELIZER, Carlos Antônio Albuquerque ${ }^{1}$, ESPÓSITO, Mario Pinheiro ${ }^{2}$, PEREIRA

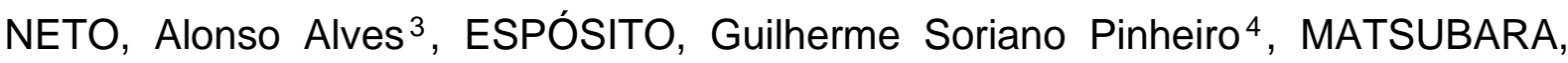
Edinéia Miyuki ${ }^{5}$

PELIZER, Carlos Antônio Albuquerque. Et al. Papiloma invertido - um relato de caso. Revista Científica Multidisciplinar Núcleo do Conhecimento. Ano. 07, Ed. 01, Vol. 03, pp. 21-27. Janeiro de 2022. ISSN: 2448-0959, Link de acesso: https://www.nucleodoconhecimento.com.br/saude/papiloma-invertido, $\quad$ DOI: 10.32749/nucleodoconhecimento.com.br/saude/papiloma-invertido

\section{RESUMO}

Este relato de caso visa apresentar um caso clínico de um paciente atendido no Hospital de Otorrino em Cuiabá- MT devido a uma queixa de cefaleia, rinorreia, obstrução nasal e roncos, abordando como foi o atendimento otorrinolaringológico e as condutas tomadas referente ao quadro, sendo realizado exames complementares e proposta terapêutica. Dessa forma, o presente estudo tem por objetivo relatar um caso de Papiloma Invertido - PI em jovem do sexo masculino, como foi feita a avaliação otorrinolaringológica e as propostas de tratamento, a fim de fomentar as discussões sobre obstrução nasal e o manejo clínico do PI. O paciente foi submetido a cirurgia para exérese da lesão, constatando, através de estudo anatomopatológico, ser Papiloma Invertido (PI). Por fim, concluímos que todo quadro

\footnotetext{
${ }^{1}$ Residente em Otorrinolaringologia e Cirurgia Cérvico-Facial; Graduado em Medicina pelo Centro Universitário São Lucas - UNISL.

${ }^{2}$ Doutor em Otorrinolaringologia pela Faculdade de Ciências Médicas da Santa Casa de São Paulo.

${ }^{3}$ Residente em Otorrinolaringologia e Cirurgia Cérvico-Facial, Graduado em Medicina pela União das Faculdades dos Grandes Lagos - UNILAGO.

${ }^{4}$ Residente em Otorrinolaringologia e Cirurgia Cérvico-Facial; Graduado em Medicina pelo Centro Universitário São Lucas - UNISL.

${ }^{5}$ Graduanda em Medicina pela Universidade de Cuiabá - UNIC.
}

RC: 104900

Disponível em: https://www.nucleodoconhecimento.com.br/saude/papiloma-invertido 
de obstrução nasal deve ser investigado e avaliado por um especialista, e ter o PI como uma possibilidade diagnóstica.

Palavras-chave: Papiloma Invertido, Obstrução nasal, Tumor nasal.

\section{INTRODUÇÃO}

Em 1854, Ward descreveu o primeiro tipo de tumor em cavidade nasal conhecido como Papiloma Invertido (PI), conforme descrito por Salomone et al. (2008). Esse termo, refere-se a uma neoplasia benigna de origem no epitélio de revestimento, surgindo, dessa forma, na mucosa respiratória que reveste a cavidade nasal e os seios paranasais, pode-se acometer na parede lateral e no meato médio nasal, também conhecido como membrana de Schneider. De acordo com Gu; Wang; Cao et al. (2015, n.p.), "Inverted papillomas (IPs) are common benign sinonasal tumors characterized by a high risk of recurrence and potential for malignancy"[6]. Entendemos, portanto, pesquisando na literatura que são raras as vezes que se encontram no septo nasal e nos seios paranasais.

A incidência do PI ocorre em torno de 1,5 caso por 100.000 habitantes e corresponde 0,5 a $4 \%$ de todos os tumores nasais, tendo o gênero mais acometido o sexo masculino entre a $5^{\underline{a}}$ e $6^{\underline{a}}$ década de vida, de acordo com Batsakis (1979). As manifestações clínicas não são bem definidas, entretanto pacientes relatam, em sua maioria, obstrução nasal unilateral por longos períodos ou sintomas inespecíficos como: rinorreia, cefaleia, hiposmia, rinossinusites, epistaxe e distúrbio olfatório, conforme citado por Salomone et al. (2008).

Histologicamente, o PI costuma ser caracterizado ser um tumor benigno, de origem ectodérmica, segundo Salomone et al. (2008). O PI, entretanto, é capaz de transpassar as paredes ósseas dos seios paranasais, podendo se alocar, também, nos tecidos moles circunvizinhos. Salomone et al. (2008), compreendem que

[...] apesar de benigno, o papiloma invertido caracteriza-se pelo crescimento agressivo, grande potencial de invasão multicentrismo (12\%), índices elevados de recidiva e malignização (2 a 53\%). Cerca de $10 \%$ dos casos de 
PI com presença de atipia celular tem associação com carcinoma de células escamosas. (SALOMONE et al., 2008, n.p.)

De acordo com Lawson; Shaari; Biller (1995), quando o PI se aloca nos tecidos moles circunvizinhos a taxa de malignamente varia de 0 a $78 \%$ e possui elevada recidiva.

A etiologia do PI ainda não é elucidada. A literatura crê que o Papiloma Vírus Humano (HPV) é um dos agentes responsáveis pelo desenvolvimento deste tumor, assim como a alergia, sinusite crônica, pólipos nasais e os fatores ambientais, Salomone et al. (2008).

A origem da Pl é descrita na maioria das vezes pela parede lateral do nariz, ao nível do meato médio, com envolvimento de fossa nasal e tendo predominância de seios paranasais maxilares e etmoidais, segundo Lawson; Shaari; Biller (1995).

O diagnóstico da PI é feito pela anamnese, exame otorrinolaringológico, tomografia computadorizada e a ressonância magnética, visto que esses auxiliam na diferenciação entre outras patologias nasais e fornece com clareza a forma, o tamanho e a extensão da doença.

Essas diferenciações são realizadas com o pólipo antrocoanal, pólipo escamoso do vestíbulo nasal, displasia fibrosa e granuloma de células gigantes. O tratamento é essencialmente cirúrgico e as técnicas e vias de acesso devem ser individualizadas.

O objetivo desse artigo é relatar um caso de Papiloma Invertido - PI em jovem do sexo masculino, como foi feita a avaliação otorrinolaringológica e as propostas de tratamento, a fim de fomentar as discussões sobre obstrução nasal e o manejo clínico do Pl. Além do mais, visa discutir e avaliar os aspectos epidemiológicos e o tratamento cirúrgico desta patologia, pois de acordo com Salomone et al. (2008, n.p.):

O tratamento cirúrgico deve ser minucioso, com a realização de um estudo radiológico prévio e bem detalhado dos limites do tumor, para que possa ser realizada a melhor técnica de abordagem e assim, retirá-lo por completo e diminuir os riscos de sua recidiva. 


\section{METODOLOGIA}

O presente trabalho foi aprovado pelo Comitê de ética do Hospital Otorrino de Cuiabá - MT. O paciente em estudo deu entrada no serviço de pronto atendimento do Hospital Otorrino de Cuiabá - MT, em 17 de dezembro de 2019 com queixa de cefaleia, rinorreia, obstrução nasal e roncos. Este foi submetido a anamnese completa, exame físico da orofaringe, cavidade nasal e ouvidos, incluindo exames de videonasofibroscopia e exames radiológicos, constatando-se tratar de um caso de Papiloma Invertido.

Para fundamentar a discussão, realizou-se também uma revisão da literatura sobre Papiloma Invertido.

\section{RELATO DE CASO}

Paciente G. E. S. de 23 anos, sexo masculino, veio no serviço de pronto atendimento do Hospital Otorrino de Cuiabá - MT, em 17 de dezembro de 2019. O paciente queixava-se de cefaleia frontal, rinorreia mucoide, obstrução nasal e roncos de longa data, nega queixas de tosse, febre, prostração e queda do estado geral. Ao ser submetido ao exame otorrinolaringológico não apresentou alterações em cavidade oral e, também, não possuía alterações em otoscopia, porém, a rinoscopia apresenta hipertrofia de cornetos inferiores, desvio septal e tumoração em cavidade nasal a direita. Como complementação dos exames, foi solicitado uma videonasofibroscopia que evidenciou a presença de tumoração polipoide em toda a cavidade direita, portanto, foi realizada uma tomografia computadorizada para avaliação de inserção e extensão da massa tumoral. O paciente foi submetido a cirurgia para correção de deformidade condrovomeriana associado a turbinectomia bilateral e sinusectomia maxilo-etmoidal bilateral e ressecção do tumor. Após 30 dias de pós-operatório, ele retorna para reavaliação em que nega quaisquer queixas, relata melhora do quadro obstrutivo e melhor qualidade de sono, o exame anatomopatológico identifica ser papiloma invertido sem alterações para malignidade. O paciente, no momento, segue sem queixas e sob acompanhamento ambulatorial. 


\section{DISCUSSÃO}

O papiloma invertido $(\mathrm{PI})$ é um tumor de origem ectodérmica, se origina na mucosa nasal e nos seios paranasais, em geral o PI é um tumor benigno. Esse tumor acomete homens, durante sua quinta e sexta década de vida, como demonstra a literatura de acordo com Lawson; Shaari; Biller (1995). De acordo com o estudo de Chee e Sethi (1999), conseguiu-se estabelecer uma média de incidência do PI, resultando dessa forma, uma média de $78 \%$ de acometimento em pacientes do sexo masculino e $22 \%$ no sexo feminino.

Esse tumor é constantemente encontrado acoplado a parede nasal lateral, podendo se infiltrar no seio paranasais ou estruturas adjacentes. Segundo estudos de Gu; Wang; Cao et al. (2015), 62,5\% dos casos de PI se originam na parede nasal lateral.

O PI apresenta-se na quinta ou sexta década de vida e é predominantemente encontrada no sexo masculino (BATSAKIS, 1979). Todavia, este relato de caso demonstra a necessidade de investigação para todas as queixas de obstrução nasal de longa data e, consequentemente, é necessário sempre suspeitar e investigar a presença de Papiloma Invertido.

É necessário que o médico otorrinolaringologista faça uma anamnese completa e um exame físico minucioso em todos seus pacientes, e em especial aqueles que recorrem com frequência as unidades de atendimento relatando que não houve uma melhora do quadro evolutivo, deve-se lançar mão de exames radiológicos e imagem que possibilitam melhor elucidação do diagnóstico.

Toda suspeita de PI deve ser investigada com biópsia da tumoração, pois seu tratamento é cirúrgico, não apresentando bons resultados para radioterapia ou quimioterapia. Para tanto, uma avaliação cirúrgica é fundamental. É necessário um exame de videonasoscopia e tomografia computadorizada para avaliar as particularidades do pólipo como extensão, local de inserção, densidade, se há penetração em outras cavidades ou tecidos. 
O método cirúrgico deve ser individualizado para cada paciente, de acordo com Marinho et al. (2010), em seu artigo "Papiloma invertido: impacto da escolha da abordagem cirúrgica e importância do surgimento pós-operatório" afirma que: "o papiloma invertido é uma lesão agressiva e recidivante, que deve ser abordada cirurgicamente conforme a extensão e localização tumoral, obedecendo às particularidades de cada caso" (MARINHO et al., 2010, p. 195).

O pós-cirúrgico deve ser submetido a um acompanhamento de longo prazo devido à alta taxa de recorrência do $\mathrm{PI}$, que corresponde a $16 \%$, de acordo com Lawson; Shaari; Biller (1995).

\section{CONCLUSÃO}

Baseado na literatura e no desfecho do relato de caso apresentado é mister afirmar que todo quadro de obstrução nasal deve ser investigado e avaliado por um especialista, e ter o PI como uma possibilidade diagnóstica.

É necessário concluir que o PI deve possuir um diagnóstico distinto de todo quadro de obstrução nasal associado a massa indeterminada em cavidade nasal. Ao ser confirmada tal patologia a conduta e tratamento são baseados em remoção cirúrgica individualizado para cada paciente, afinal a abordagem cirúrgica depende do tamanho da lesão, e podem ser lançadas alternativas de eletrodissecação, criocirurgia e laser afim de melhorar o prognostico pós-operatório segundo Marinho et al. (2010).

\section{REFERÊNCIAS}

BATSAKIS, J. G. Tumors of the Head and Neck. Baltimore. The Williams \& Wilkins Company, 1979.

CHEE, L. W. J.; SETHI, D.S. The Endoscopic Management Of Sinosal Inverted Papillomas. Clin. Otolaryngol. 24:61-6, 1999. Disponível em: < 
https://onlinelibrary.wiley.com/doi/abs/10.1046/j.1365-2273.1999.00222.x>. Acesso em: 10 de agost. 2021.

GU, Z. W.; WANG, Y. X.; CAO, Z. W. Inverted papilloma originating from the left ethmoid sinus invading the nasal cavity bilaterally via the frontal sinus septum. São Paulo, 2015. Vol. 81, №6. Disponível em: https:/www.scielo.br/scielo.php?script=sci_arttext\&pid=S1808$86942015000600684 \&$ lang=pt>. Acesso em: 22 de julh. 2020.

LAWSON, W., SHAARI, B. T. H. O., BILLER, H. F. Inverted papilloma: a report of 112 cases. Wiley Online Library, $1995 . \quad$ Disponível em: https://pubmed.ncbi.nlm.nih.gov/7877417/>. Acesso em: 22 de julh. 2020.

MARINHO, M.; GONZAGA, M.; PASCOAL, G.; PAIVA, T.; MARINHO, E.. Papiloma invertido: impacto da escolha da abordagem cirúrgica e importância do seguimento pós-operatório. Rev. Bras Cir Craniomaxilofac, 2010. Disponível em: <http://www.abccmf.org.br/cmf/Revi/2010/dezembro/15-Papiloma\%20invertido.pdf>. Acesso em: 22 de julh. 2020.

SALOMONE, R.; MATSUYAMA, C.; GIANNOTTI, O.; ALVARENGA, M.; MARTINEZ, E.; CHAVES, A. Papiloma invertido bilateral: relato de caso e revisão da literatura. Ver. Bras. Otorrinolaringol. São Paulo, 2008. Disponível em: <https://www.scielo.br/scielo.php?script=sci_arttext\&pid=S003472992008000200022>. Acesso em: 22 de julh. 2020.

\section{APÊNDICE - REFERÊNCIA NOTA DE RODAPÉ}

6. Os papilomas invertidos (IPs) são tumores nasossinusais benignos comuns, caracterizados por um alto risco de recorrência e potencial para malignidade. (Tradução nossa)

Enviado: Dezembro, 2021.

Aprovado: Janeiro, 2022. 